\title{
Prognostic and predictive biomarkers in nonmetastatic colorectal cancers
}

\author{
Fady GH Haddad*,1, Roland Eid¹, Hampig Raphael Kourie¹, Elie Barouky² \& Marwan Ghosn ${ }^{1}$ \\ ${ }^{1}$ Hematology-Oncology Department, Faculty of Medicine, Saint Joseph University, Beirut, Lebanon \\ ${ }^{2}$ General Surgery Department, Faculty of Medicine, Saint Joseph University, Beirut, Lebanon \\ *Author for correspondence: fadyhaddad.fh@gmail.com
}

\author{
"Numerous molecules and factors were recently studied as prognostic biomarkers in \\ nonmetastatic colon cancer. These include HER2, POLE, CDX2 and circulating tumor DNA, with \\ different specific implications."
}

First draft submitted: 23 January 2018; Accepted for publication: 10 April 2018; Published online: 13 August 2018

Keywords: adjuvant • biomarkers $\bullet$ CDX2 • CMS • colorectal cancer • microsatellite instability • mismatch repair • nonmetastatic $\bullet$ novel biomarkers $\bullet$ prognostic

Colorectal cancer (CRC) is the third most commonly diagnosed cancer worldwide. Surgery alone or combined with adjuvant chemotherapy remains the cornerstone of the treatment of nonmetastatic CRC [1]. The administration of adjuvant chemotherapy depends mainly on the disease stage, which is considered the strongest prognostic factor. Recently, different biological and molecular factors have been proposed as prognostic tools, used for the selection of patients that would derive a benefit from adjuvant treatment [2].

Three molecular targets represent independent prognostic biomarkers in CRC and are the most tested: mismatch repair (MMR) protein expression, $K R A S$ and $B R A F$ genes and their mutations [3-5]. At present, the assessment of the mutational status of $R A S$ and $B R A F$ is accepted as a standard practice in the management of patients with metastatic CRC, and helps determine, which patient would benefit from an EGFR inhibitor therapy as well as the prognosis [6]. Thus for now, the adequate molecular profiling of patients with CRC involves assessment of tumor genomic profiling for $K R A S, N R A S$, and $B R A F$ along with DNA MMR status by immunohistochemistry or microsatellite instability (MSI) screening.

We aim here to focus on some new potential prognostic and predictive biomarkers that will help stratify patients, along with a potential role in determining the utility of an adjuvant treatment in nonmetastatic colorectal cancers.

\section{MMR status \& implications}

MMR status plays a role as a prognostic factor and as a predictive marker for adjuvant chemotherapy in patients with stage II and III CRC [7]. First, CRC with a high MSI level (MSI-H) or deficient MMR status draws modest, if no benefit, from fluorouracil-based (FU) chemotherapy indicating the need for risk-stratifying predictive markers. Second, dMMR CRC shows a more favorable prognosis than proficient MMR (pMRR), supporting the strategy of withholding adjuvant chemotherapy in this setting with observation; thereby sparing patients toxicities related to chemotherapy [8]. Of note, is that these conclusions are derived mostly from retrospective studies in which tumor stage and patient age could have influenced treatment strategy. As such, fit patients with stage III CRC are treated with adjuvant chemotherapy regardless of MMR status. On the other hand, MMR status had a significant impact on treatment for young patients with stage II tumors, while in elderly patients, age seemed to become the major determinant factor for the use of adjuvant chemotherapy [9].

Preliminary data showed that the resistance to FU/leucovorin in dMMR patients may be overcome by the addition of oxaliplatin or irinotecan to FU/leucovorin, the standard treatment regimen for stage III CRC [10,11]. However, more recent results from the PETACC-3 trial found that the effect of MSI status on relapse-free survival (RFS) and overall survival (OS) was stronger in stage II than in stage III patients, with superiority to MSI-H over 
microsatellite stable (MSS) tumors. In addition, and contrarily to what has been described earlier, the treatment with FOLFIRI was not superior to the 5-FU/leucovorin, suggesting no additional benefit from the addition of irinotecan in MSI-H tumors [12].

As such, the patients with stage II CRC that are considered for single-agent fluoropyrimidine-based treatment should have the tumor MMR status assessed to inform the likelihood of patient benefit of chemotherapy and to guide decision as to which patients should not be offered adjuvant treatment (deficient MMR vs pMRR) [8]. Of note, is that the frequency of dMMR tumors decreases with advancing stage, ranging from 22 to $12 \%$ and $3 \%$ in stage II, III and IV, respectively [13].

The patients with dMMR have ten- to 100-times more somatic mutations than pMMR. Moreover, dMMR cancers are infiltrated by lymphocytes, a finding consistent with an immune response [14]. Consequently, dMMR tumors are found to be more responsive to immunotherapy, particularly PD-1 blockade. In fact, pembrolizumab has recently received an accelerated approval for the treatment of patients with unresectable or metastatic solid tumors, with a high level of MSI or dMMR that have progressed after prior treatment, notably with fluoropyrimidine, oxaliplatin, and irinotecan in the case of CRC. In the setting of CRC, the objective response rate was shown to be $36 \%$ [15]. PD-L1 inhibition is also being evaluated in early stage MSI-H colon cancer, with the Alliance A021502 trial planning to evaluate the combination of FOLFOX \pm atezolizumab in the adjuvant setting of stage III colon cancer, with the primary end point being disease-free survival (DFS) [13].

\section{MMR \& BRAF}

CRC is also characterized by the presence of BRAF V600E mutation in nearly $10 \%$ of cases, which is mutually exclusive of the KRAS mutations found in $40-45 \%$ of cases. BRAF mutation is associated with female sex, proximal tumors, older age, high grade and MSI-H tumors [16]. The presence of the BRAF V600E mutation is interpreted along with the MMR status in patients with stage III colon cancer to allow a precise stratification of patients and to identify those with poor clinical outcomes. In fact, in MSS tumors, the BRAF mutation is associated with a shorter DFS and OS. On the other hand, this mutation was linked to an improved and longer DFS in tumors with MSI-H, but OS was not significantly affected. Thus, in stage III colon cancers, patients with MSI-H/BRAF wild-type profile had the best prognosis, compared with the worst prognosis observed in patients with MSS/BRAF V600E mutation. Intermediate survival was noted in patients with MSI-H/BRAF V600E mutation and MSS/BRAF wildtype tumors [17]. Along with its established role as a prognostic biomarker, $B R A F$ mutation seems to progressively gain place as a therapeutic target, yet in the metastatic but not adjuvant setting. In fact, targeting BRAF will also require the blockade of other pathways including, among others, EGFR, MEK and PI3K [18].

\section{MMR \& KRAS}

The analysis of the relationship between the KRAS and MMR status showed that the presence of KRAS mutations was associated with a shorter DFS and OS in patients with MSS tumors which represent nearly $90 \%$ of all stage III colon cancer; whereas in tumors with MSI-H, this effect was not present with no implication of KRAS mutations on survival [19]. In addition, MSS tumors are located in the distal colon segment in the majority of cases, whereas MSI-H tumors are mainly proximal [20]. This observation is concordant with recent data showing that mutations in codons 12 and 13 of the KRAS gene were associated with a worse prognosis only in patients having a distal tumor [17,21]. Meanwhile, the predictive effect of KRAS mutations on survival in stage II/III colon cancer remains controversial with conflicting evidence regarding the impact of this mutation on recurrence and OS [22].

\section{Novel biomarkers}

Numerous molecules and factors were recently studied as prognostic biomarkers in nonmetastatic colon cancer. These include HER2, POLE, CDX2 and circulating tumor DNA, with different specific implications.

\section{HER2/Erbb2}

Another prognostic biomarker is the HER2/Erbb2 protein. Although rare, occurring in less than $4 \%$ of patients with stage III colon cancer, Erbb2 alterations are associated with a worse prognosis [23]. Erbb2 alterations comprised amplifications and mutations, the later accounting for a quarter of all alterations in this gene. The occurrence of Erbb2 mutations was found to be independent of RAS or BRAF and was not mutually exclusive.

Erbb2 alterations indicated a poorer prognosis with a reduced OS and a shorter time to recurrence. Thus, these alterations emerged as novel targets in metastatic colorectal cancer (mCRC), supported by the response to 
dual-targeted therapy with trastuzumab and lapatinib in patients with HER2-positive mCRC [24]. These findings lead to the consideration of Erbb2 testing along with adjuvant strategies [23]. However, with the emerging role of next-generation sequencing in the detection of molecular alterations notably in CRC, via gene panels allowing the study of a larger number of genetic alterations at lower costs [25], Erbb2 could become included in future standardized sequencing panels.

\section{DNA polymerase epsilon}

DNA polymerase epsilon (POLE)-mutated CRCs are exceptionally mutated (ultra-mutated) cancers caused by mutations that impair POLE proofreading. They represent around $1 \%$ of all CRCs. The patients carrying POLE mutations are younger at diagnosis, more frequently men and more frequently diagnosed with right-sided colorectal tumors. POLE-mutated CRCs displayed increased $\mathrm{CD}^{+}$lymphocyte infiltration and expression of cytotoxic Tcell markers and effector cytokines, similar in extent to that observed in immunogenic dMMR cancers [26]. POLE proofreading domain mutations identify a subset of immunogenic colorectal cancers with excellent prognosis. What may be of greatest interest is the therapeutic potential for immune checkpoint blockade in the presence of POLE mutations in metastatic CRC, in which single agent PD-1 blockade has been largely ineffective in dMMR tumors [27]. Therefore, given the high mutational load in POLE tumors and the upregulation of immune checkpoints, these tumors may represent a perfect target for immunotherapy and need to be further investigated. In fact, future trials will be evaluating the role of immune checkpoint inhibitors (e.g., the anti-PD-L1 agent avelumab) in the treatment of POLE-mutated CRC [28,29].

\section{Homeobox transcription factor}

The caudal-type homeobox transcription factor 2 (CDX2) was identified as a candidate biomarker of mature colon epithelial tissues. It is detected by immunohistochemistry techniques using the antihuman CDX2 monoclonal antibody on formalin-fixed, paraffin-embedded tissue sections. Around $13 \%$ of colon cancer did not express the CDX2. A recent study showed that lack of CDX2 expression is correlated with high-risk stage II colon cancer that may probably benefit from adjuvant chemotherapy [30].

Further data suggested that although globally CDX2 expression was considered an independent prognostic factor, its prognostic effect varied according to consensus molecular subtypes (CMS) of colon cancer; with CMS1 being characterized by MSI-H, strong immune activation and good prognosis (14\% of CRC), compared with CMS4 showing a mesenchymal/stem cell phenotype, a strong activation of the TGF- $\beta$ pathway, stromal invasion, angiogenesis and a poor prognosis (23\% of CRC) [31,32]. The examination of the prognostic impact of CDX2 separately in CMS1 and CMS4 showed that CDX2 status was not prognostic in CMS1, while it was highly prognostic in CMS4 tumors for both RFS and OS. Moreover, the comparison between MSS and MSI-H found that CDX2 status was prognostic in MSS patients both for OS and RFS, but not prognostic in MSI-H patients [31]. Thus, CMS and CDX2 may play an important role as stratification factors for future adjuvant trials.

\section{Circulating tumor DNA}

An alternative strategy to standard analyses of the resected surgical specimen is to directly examine plasma for evidence of residual disease. Circulating tumor DNA (ct-DNA) has been shown to be a promising blood biomarker in metastatic CRC. However, in nonmetastatic CRC, particularly in postoperative resection of stage II colon cancer, early data suggest that ct-DNA provides evidence of residual disease and is considered a promising marker for identifying patients at high risk of recurrence; with a shorter recurrence-free survival in patients with detectable ct-DNA [33].

\section{PI3K mutations \& the role of adjuvant salicylic acid}

$\mathrm{PI} 3 \mathrm{~K} / \mathrm{AKT}$ is an intracellular signaling pathway that leads to apoptosis, stimulates growth and increases proliferation. Various changes in the PI3K/AKT pathway are observed in around $40 \%$ of CRC, such as disruptions of KRAS, PIK3CA and PTEN mutations, and IGF2 overexpression [34]. These mutations were studied to determine if they had any prognostic impact. Of note, mutations in PIK3CA are present in 10-15\% of CRC and are correlated with proximal colonic tumors, mucinous differentiation, KRAS mutation and were associated with adverse outcomes for patients with BRAF wild-type tumors [35]. In patients with stage III colon cancer, PIK3CA mutations in the exon 20 have been suggested to have a negative impact on prognosis [36], similarly to their unfavorable effect on PFS and OS in the metastatic setting of patients treated with cetuximab [37]. In addition, patients harboring 
both exon 9 and 20 mutations exhibit a worse prognosis compared with patients with PIK3CA wild-type or with PIK3CA mutation in either of the exons [38].

In contrast, some reports have suggested that PIK3CA mutations are associated with longer survival and reduced rate of recurrence for patients that use aspirin (salicylic acid) regularly after diagnosis [39]. This benefit from the use of aspirin after CRC diagnosis is observed among patients with PIK3CA mutations but not among patients with wild-type PIK3CA tumors, with studies showing a 29\% mortality reduction in PIK3CA mutant disease. These findings suggest that PIK3CA mutation in CRC could serve as a predictive molecular biomarker for adjuvant aspirin therapy, although more trials are needed to evaluate the efficacy of aspirin in the adjuvant setting [40,41].

\section{Future perspective}

In this era of precision cancer medicine, molecular biomarkers are being investigated in order to be implemented in clinical practice. In the nonmetastatic setting, prognostic biomarkers are needed to help stratify patients and predict survival. Of these biomarkers, MMR status, RAS, RAF, HER2, POLE, CDX2 along with others help predicting a specific prognosis for patients with CRC. These biomarkers could serve in the future as predictive factors for response to certain adjuvant chemotherapies. It may also be interesting in the future that a molecular biomarker could be incorporated into a predictive score model determining the benefit from adjuvant chemotherapy.

Financial \& competing interests disclosure

The authors have no relevant affiliations or financial involvement with any organization or entity with a financial interest in or financial conflict with the subject matter or materials discussed in the manuscript. This includes employment, consultancies, honoraria, stock ownership or options, expert testimony, grants or patents received or pending, or royalties.

No writing assistance was utilized in the production of this manuscript.

\section{References}

Papers of special note have been highlighted as: $\bullet$ of interest; $\bullet \bullet$ of considerable interest

1. Gustavsson B, Carlsson G, Machover D et al. A review of the evolution of systemic chemotherapy in the management of colorectal cancer. Clin. Colorect. Cancer 14(1), 1-10 (2015).

2. Rodrigues D, Longatto-Filho A, Martins SF. Predictive biomarkers in colorectal cancer: from the single therapeutic target to a plethora of options. BioMed Res. Int. 2016 (2016).

- It is very important to always study molecular biomarkers and incorporate them in clinical practice as prognostic of predictive tools.

3. Fariña-Sarasqueta A, van Lijnschoten G, Moerland $\mathrm{E}$ et al. The BRAF V600E mutation is an independent prognostic factor for survival in stage II and stage III colon cancer patients. Ann. Oncol. 21(12), 2396-2402 (2010).

4. Lièvre $\mathrm{A}$, Bachet $\mathrm{J}-\mathrm{B}$, Boige $\mathrm{V}$ et al. KRAS mutations as an independent prognostic factor in patients with advanced colorectal cancer treated with cetuximab. J. Clin. Oncol. 26(3), 374-379 (2008).

5. Ohrling K, Edler D, Hallström M, Ragnhammar P. Mismatch repair protein expression is an independent prognostic factor in sporadic colorectal cancer. Acta Oncol. Stockh. Swed. 49(6), 797-804 (2010).

6. Shen Y, Han X, Wang J et al. Prognostic impact of mutation profiling in patients with stage II and III colon cancer. Sci. Rep. 6, 24310-24317 (2016).

7. Sinicrope FA. DNA mismatch repair and adjuvant chemotherapy in sporadic colon cancer. Nat. Rev. Clin. Oncol. 7(3), 174-177 (2010).

8. Sargent DJ, Marsoni S, Monges G et al. Defective mismatch repair as a predictive marker for lack of efficacy of fluorouracil-based adjuvant therapy in colon cancer. J. Clin. Oncol. 28(20), 3219-3226 (2010).

9. He EY, Hawkins NJ, Mak G et al. The impact of mismatch repair status in colorectal cancer on the decision to treat with adjuvant chemotherapy: an Australian population-based multicenter study. Oncologist 21(5), 618-625 (2016).

10. Zaanan A, Cuilliere-Dartigues P, Guilloux A et al. Impact of p53 expression and microsatellite instability on stage III colon cancer disease- free survival in patients treated by 5 -fluorouracil and leucovorin with or without oxaliplatin. Ann. Oncol. 21(4), 772-780 (2010).

11. Bertagnolli MM, Niedzwiecki D, Compton CC et al. Microsatellite instability predicts improved response to adjuvant therapy with irinotecan, fluorouracil, and leucovorin in stage III colon cancer: Cancer and Leukemia Group B Protocol 89803. J. Clin. Oncol. 27(11), 1814-1821 (2009)

12. Klingbiel D, Saridaki Z, Roth AD, Bosman FT, Delorenzi M, Tejpar S. Prognosis of stage II and III colon cancer treated with adjuvant 5-fluorouracil or FOLFIRI in relation to microsatellite status: results of the PETACC-3 trial. Ann. Oncol. 26(1), 126-132 (2015)

- With the presence of conflicting evidence, it is important to better characterize the effect of mismatch repair status on the response to chemotherapy.

13. Boland PM, Ma WW. Immunotherapy for colorectal cancer. Cancers 9(5), 50-62 (2017). 
14. Kim ST, Klempner SJ, Park SH et al. Correlating programmed death ligand 1 (PD-L1) expression, mismatch repair deficiency, and outcomes across tumor types: implications for immunotherapy. Oncotarget 8(44), 77415-77423 (2017).

15. Broderick JM. FDA approves pembrolizumab for microsatellite instability-high and mismatch repair deficient cancers. OncLive, 23rd May (2017).

16. Roth AD, Tejpar S, Delorenzi M et al. Prognostic role of KRAS and BRAF in stage II and III resected colon cancer: results of the translational study on the PETACC-3, EORTC 40993, SAKK 60-00 trial. J. Clin. Oncol. 28(3), 466-474 (2010).

17. Taieb J, Zaanan A, Le Malicot $\mathrm{K}$ et al. Prognostic effect of BRAF and KRAS mutations in patients with stage III colon cancer treated with leucovorin, fluorouracil, and oxaliplatin with or without cetuximab: a post hoc analysis of the PETACC-8 trial. JAMA Oncol. 14, $1-11(2016)$.

18. Jones JC, Grothey A. Understanding BRAF-mutant colorectal cancer. ASCO Daily News, 26th May (2016). https://am.asco.org/daily-news/understanding-braf-mutant-colorectal-cancer

19. Phipps AI, Buchanan DD, Makar KW et al. KRAS-mutation status in relation to colorectal cancer survival: the joint impact of correlated tumour markers. Br. J. Cancer 108(8), 1757-1764 (2013).

20. $\mathrm{Hu}$ J, Yan W-Y, Xie L et al. Coexistence of MSI with KRAS mutation is associated with worse prognosis in colorectal cancer. Medicine (Baltimore) 95(50), (2016).

21. Blons H, Emile JF, Le Malicot K et al. Prognostic value of KRAS mutations in stage III colon cancer: post hoc analysis of the PETACC8 Phase III trial dataset. Ann. Oncol. 25(12), 2378-2385 (2014).

22. Kim HS, Heo JS, Lee J et al. The impact of KRAS mutations on prognosis in surgically resected colorectal cancer patients with liver and lung metastases: a retrospective analysis. BMC Cancer 16, 120 (2016).

23. Laurent-Puig P, Balogoun R, Cayre A et al. ERBB2 alterations a new prognostic biomarker in stage III colon cancer from a FOLFOX based adjuvant trial (PETACC8). Ann. Oncol. 27(Suppl. 6), 459 (2016).

24. Sartore-Bianchi A, Trusolino L, Martino C et al. Dual-targeted therapy with trastuzumab and lapatinib in treatment-refractory, KRAS codon 12/13 wild-type, HER2-positive metastatic colorectal cancer (HERACLES): a proof-of-concept, multicentre, open-label, Phase 2 trial. Lancet Oncol. 17(6), 738-746 (2016).

25. Fontanges Q, De Mendonca R, Salmon I, Le Mercier M, D'Haene N. Clinical application of targeted next generation sequencing for colorectal cancers. Int. J. Mol. Sci. 17(12), 2117-2127 (2016).

- Next-generation sequencing represents an effective and affordable technique with enormous information provided. In the future, it could replace standard techniques in the detection of genetic aberrations.

26. Domingo E, Freeman-Mills L, Rayner E et al. Somatic POLE proofreading domain mutation, immune response and prognosis in colorectal cancer: a retrospective, pooled biomarker study. Lancet Gastroenterol. Hepatol. 1, 207-216 (2016).

27. Eng C. POLE mutations in colorectal cancer: a new biomarker? Lancet Gastroenterol. Hepatol. 1(3), 176-177 (2016).

28. Ahn S-M, Ansari AA, Kim J et al. The somatic POLE P286R mutation defines a unique subclass of colorectal cancer featuring hypermutation, representing a potential genomic biomarker for immunotherapy. Oncotarget 7(42), 68638-68649 (2016).

-. With the era of immunotherapy, we are in need of new biomarkers opening the field for new checkpoint inhibitors indications thus better responses, hopefully.

29. Nebot-Bral L, Brandao D, Verlingue L et al. Hypermutated tumours in the era of immunotherapy: the paradigm of personalised medicine. Eur. J. Cancer 84, 290-303 (2017).

30. Dalerba P, Sahoo D, Paik S et al. CDX2 as a prognostic biomarker in stage II and stage III colon cancer. N. Engl. J. Med. 374(3), 211-222 (2016).

31. Pilati C, Taieb J, Balogoun R, Marisa L, de Reyniès A, Laurent-Puig P. CDX2 prognostic value in stage II/III resected colon cancer is related to CMS classification. Ann. Oncol. 28(5), 1032-1035 (2017).

- CDX2 represents a prognostic biomarker that is not well known, but merits further studies due to the various prognostic effects it could hold, especially in association with consensus molecular subtypes.

32. Guinney J, Dienstmann R, Wang X et al. The consensus molecular subtypes of colorectal cancer. Nat. Med. 21(11), 1350-1356 (2015).

-. The consensus molecular subtypes offer a new and more comprehensive description of the various types of colorectal cancer.

33. Tie J, Wang Y, Tomasetti $\mathrm{C}$ et al. Circulating tumor DNA analysis detects minimal residual disease and predicts recurrence in patients with stage II colon cancer. Sci. Transl. Med. 8(346), 346ra92 (2016).

- Circulating tumor DNA is a promising tool for many tumor types, and could largely replace tissue biopsies in the coming years.

34. Danielsen SA, Eide PW, Nesbakken A, Guren T, Leithe E, Lothe RA. Portrait of the PI3K/AKT pathway in colorectal cancer. Biochim. Biophys. Acta 1855(1), 104-121 (2015).

35. Rosty C, Young JP, Walsh MD et al. PIK3CA activating mutation in colorectal carcinoma: associations with molecular features and survival. PLoS ONE 8(6), e65479 (2013).

36. Fariña Sarasqueta A, Zeestraten ECM, van Wezel T et al. PIK3CA kinase domain mutation identifies a subgroup of stage III colon cancer patients with poor prognosis. Cell. Oncol. Dordr. 34(6), 523-531 (2011). 
37. De Roock W, Claes B, Bernasconi D et al. Effects of KRAS, BRAF, NRAS, and PIK3CA mutations on the efficacy of cetuximab plus chemotherapy in chemotherapy-refractory metastatic colorectal cancer: a retrospective consortium analysis. Lancet Oncol. 11(8), 753-762 (2010).

38. Liao X, Morikawa T, Lochhead P et al. Prognostic role of PIK3CA mutation in colorectal cancer: cohort study and literature review. Clin. Cancer Res. 18(8), 2257-2268 (2012).

39. Domingo E, Church DN, Sieber O et al. Evaluation of PIK3CA mutation as a predictor of benefit from nonsteroidal anti-inflammatory drug therapy in colorectal cancer. J. Clin. Oncol. 31(34), 4297-4305 (2013).

40. Liao X, Lochhead P, Nishihara R et al. Aspirin use, tumor PIK3CA mutation, and colorectal-cancer survival. N. Engl. J. Med. 367(17), 1596-1606 (2012).

41. Paleari L, Puntoni M, Clavarezza M, DeCensi M, Cuzick J, DeCensi A. PIK3 CA mutation, aspirin use after diagnosis and survival of colorectal cancer. A systematic review and meta-analysis of epidemiological studies. Clin. Oncol. (R. Coll. Radiol.) 28(5), 317-326 (2016).

- The emerging effect of aspirin is interesting to be considered for future adjuvant management of colon cancer. 